\title{
Estructura de la comunidad íctica del arroyo Paraíso afluente del río Uruguay. Misiones. Argentina
}

\author{
Silvia Alicia Flores, Patricia Raquel Araya, Marcelo Javier Serrano, Adriana Griselda Barboza \\ Y LOURDES MARÍA HIRT \\ Facultad de Ciencias Exactas, Químicas y Naturales. Universidad Nacional de Misiones. Misiones, Argentina.
}

E-mail: silvialiciaflores@gmail.com

\begin{abstract}
RESUMEN. Se determinó la composición, diversidad específica, abundancia, número de endemismos y estructura trófica de la comunidad íctica del arroyo Paraíso, afluente del río Uruguay. Se realizaron cuatro campañas de pesca experimental entre setiembre de 2013 y marzo de 2015, utilizando ocho redes de espera monofilamento. La captura total fue de 217 individuos pertenecientes a 35 especies de las cuales el 77\% corresponde a los órdenes Characiformes (16 especies) y Siluriformes (11 especies) y el 33\% a los órdenes Cichliformes (siete especies) y Gymnotiformes (una especie). La diversidad alfa de número efectivo fue de 16,44. De acuerdo con el estimador no paramétrico Chao 1, el inventario de peces es incompleto, restarían encontrar al menos 16 especies. Las especies raras o accidentales representaron el 66\%, con abundancia relativa inferior al 10\%. Las especies comunes fueron Crenicichla missioneira, Leporinus amae, Leporinus striatus y Astyanax lacustris. Se registraron siete especies endémicas de la cuenca del río Uruguay: C. missioneira, C. celidochilus, C. tendybaguassu, L. amae, Astyanax paris, A. troya, y Hemiancistrus fulliginosus. La mayoría de las especies capturadas (83\%) fueron de pequeño porte. El índice de dominancia comunitaria fue máximo en primavera $(0,81)$. La materia orgánica de origen alóctono fue el principal recurso alimentario. Se reconocieron seis grupos tróficos y el 61,5\% de las especies perteneció al grupo de los detritívoros u omnívoros. La comunidad presentó actividad reproductiva durante todas las estaciones del año, con preponderancia en la primavera y el invierno con especies como L. striatus y A. piracicabae. La elevada diversidad específica, el endemismo y la alta dependencia del material orgánico alóctono de la ictiofauna demanda la urgente necesidad de protección y conservación de este curso de agua.
\end{abstract}

Palabras clave: Abundancia, arroyo Paraíso, biodiversidad, composición, ictiofauna neotropical.

ABSTRACT. We analyzed the composition, specific diversity, abundance, number of endemism's and trophic structure of the fish community of the Paraíso stream, affluent of the Uruguay River. Four fish samplings were carried out between September 2013 and March 2015, with eight monofilament gillnets. The total catch was 217 individuals distributed in 35 species of which $77 \%$ belong to the orders Characiformes (16 species) and Siluriformes (11 species) and the remaining 33\% to the orders Perciformes (seven species) and Gymnotiformes (one species). The alpha diversity of effective numbers was 16.44. According to the non-parametric Chao 1 estimator, the species inventory was incomplete it would be necessary to find at least 16 species. Rare or accidental species accounted for $66 \%$, with relative abundance of less than $10 \%$. Common species were Crenicichla missioneira, Leporinus amae, Leporinus striatus and Astyanax lacustris. Seven endemic species from the Uruguay River basin were registered: C. missioneira, C. celidochilus, C. tendybaguassu, L. amae, Astyanax paris, A. troya, and Hemiancistrus fulliginosus. Most of the species captured (83\%) were small. The community dominance index was higher during the spring (0.81). Organic matter of allochthonous origin was the main food resource. Six trophic categories were recognized, mostly detritivores and omnivores (61.5\%). The community presented reproductive activity during all seasons of the year, with a preponderance in spring and winter with species such as $L$. striatus and $A$. piracicabae. The 
high specific diversity, endemism and the high dependency of the allochthonous organic material of the ichthyofauna demand the urgent need of protection and conservation of this watercourse.

Keywords: Abundance, biodiversity, composition, neotropical fish fauna, Paraíso stream.

\section{INTRODUCCIÓN}

La provincia de Misiones posee un sistema hídrico muy importante, con grandes ríos como el Paraná, el Iguazú y el Uruguay. Los tributarios del río Uruguay se caracterizan por ser cortos, con saltos y cascadas (Margalot, 1985), que actúan como barreras de aislamiento, razón por la cual la presencia de especies endémicas o de distribución restringida aumenta su importancia para la conservación.

Estudios sobre la comunidad íctica de la provincia de Misiones se presentan en Gómez y Chebez (1996), Giraudo et al. (2003) y Roa (2005). Una revisión sobre la diversidad ictiofaunística de la región mesopotámica de la Argentina menciona 22 especies en tributarios del río Uruguay en Misiones (López et al., 2005). Investigaciones recientes han reportado nuevas especies que amplían la lista. Así, Rosso et al. (2013) identifican 36 especies para el sistema Ramos-Acaraguá en el centro de la provincia de Misiones y Araya et al. (2012) 65 especies en el arroyo Yabotí. La riqueza registrada en este arroyo mediante un estudio intensivo, triplica las cifras citadas para todos los tributarios del Uruguay y es un claro indicador de que los valores anteriores no reflejan la diversidad íctica de estos tributarios, la cual sigue siendo parcialmente conocida.

En el territorio misionero se han realizado, en la última década, numerosos descubrimientos de nuevas especies de peces. Tres de las especies descriptas fueron del género Crenicichla, Crenicichla tesay Casciotta \& Almirón, 2008 en el río Iguazú, Crenicichla yaha Casciotta, Almirón \& Gómez, 2006 del río Iguazú y arroyo Urugua-í y Crenicichla hu Piálek, Rícan, Casciotta \& Almirón, 2010 del Arroyo Piray Miní (Piálek et al., 2010, 2015). Almirón et al. (2015) describen a Oligosarcus amome Casciotta, Piálek, Doubnerová \& Ríčan, 2015, una nueva especie del río Uruguay. Además, suceden nuevos registros tales como Cyanocharax obi Casciotta, Almirón, Pialek \& Rícan, 2012 de tributarios del Paraná (Casciotta et al., 2012) y de especies del género Rineloricaria (Almirón et al., 2014). Muchos de estos nuevos registros son endemismos para los cursos interiores de Misiones, que ya cuenta con la más alta tasa de endemismo de todas las regiones ícticas de la Argentina (López et al., 2005, Reis, et al., 2016, Giraudo,

En Misiones quedan muchos ambientes acuáticos por estudiar, siendo los arroyos los que menos atención han recibido. El vacío de información sobre la ictiofauna de la provincia dificulta la comprensión de sus relaciones biogeográficas con otras regiones de la Argentina y de Sudamérica. Adicionalmente, el elevado grado de endemismo de la ictiofauna de los arroyos de Misiones no ha sido cuantificada adecuadamente, por lo que profundizar los inventarios de peces permitirá completar el esquema de distribución de los peces de agua dulce de la Argentina y elucidar sus relaciones biogeográficas de manera más precisa. Esta información es para ser aplicada en estrategias de conservación basadas en criterios científicos y por lo tanto más efectivas en una de las regiones de mayor diversidad y más amenazada de la Argentina y el mundo. El presente trabajo contribuirá al conocimiento de la diversidad ictiofaunística del arroyo Paraíso, afluente del río Uruguay, con el fin de determinar la composición, diversidad específica, abundancia, número de endemismos y estructura trófica. La importancia de esta subcuenca radica en que el caudal de agua que transporta y que alimenta a saltos escondidos tras los frondosos senderos de árboles centenarios que en conjunto representan un importante recurso turístico para la región es en parte el límite con la Reserva de Biosfera Yabotí (RBY). 


\section{MATERIALES Y MÉTODOS}

El arroyo Paraíso presenta una topografía ondulada con pendientes suaves a muy pronunciadas; con una extensión de $80 \mathrm{~km}$, es el límite noreste de la RBY y atraviesa la Reserva de Uso Múltiple Guaraní perteneciente a la Universidad Nacional de Misiones. Estas características del relieve y la extensión del recorrido condicionan el régimen de escurrimiento, que en esta región es muy variable e influenciado por las lluvias que en ocasiones se producen solo en determinadas subcuencas (Fontana, 2014).

Este curso de agua integra el Distrito de las Selvas Mixtas, Provincia Paranaense (Cabrera y Willink, 1973) y como los demás de la región, son de régimen subtropical con precipitaciones distribuidas a lo largo del año, con dos máximos que determinan dos crecientes anuales, una en otoño y otra en primavera (Fontana, 2014). Se realizaron cuatro campañas de pesca experimental de carácter estacional, en septiembre de 2013, abril y agosto de 2014 y marzo de 2015. Los puntos de muestreos se ubicaron en: (1) la desembocadura del arroyo Paraíso en el río Uruguay $\left(27^{\circ} 15^{\prime} 1.46^{\prime \prime} \mathrm{S}, 54^{\circ}\right.$ $2^{\prime} 15.92^{\prime \prime}$ O) y (2) en un punto a $7 \mathrm{~km}$ aguas arriba del anterior, en cercanías del Salto Paraíso (2714'3.91" S, 54 2'25.41" O) (Figura 1). La selección de los puntos de muestreo se basó en diferencias geomorfológicas y accesibilidad a los mismos.

El permiso para la pesca experimental fue otorgado por el Ministerio de Ecología y Recursos Naturales Renovables de la Provincia de Misiones por Disposición N035/2014 y 012/2015. Las capturas se realizaron con una batería de redes de espera monofilamento de 3,$4 ; 5 ; 6 ; 7 ; 8 ; 10$ y $12 \mathrm{~cm}$ entre nudos opuestos, que permanecieron caladas durante 24 horas en cada punto de muestreo y fueron revisadas cada 8 horas. Para el registro de datos ambientales de temperatura, $\mathrm{pH}$, conductividad y oxígeno. disuelto se utilizó un equipo multiparámetro HANNA HI 98129.

De cada individuo capturado se registraron los siguientes datos biométricos: longitud estándar $(\mathrm{LE}, \mathrm{cm})$, peso total y peso de los ovarios en gramos (g). Se identificó el sexo y estadio

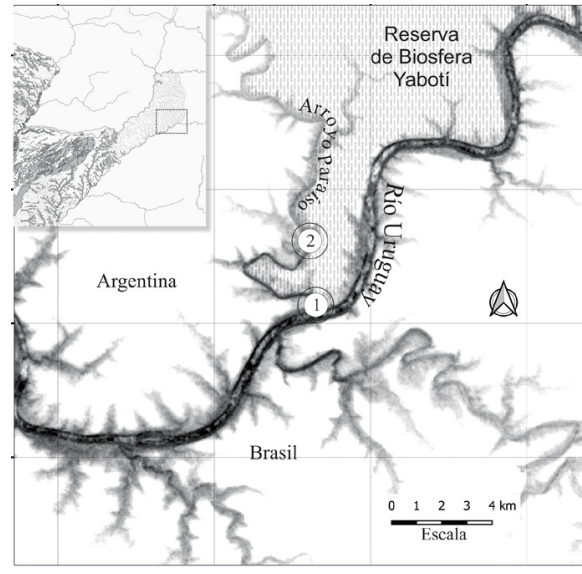

Figura 1. Sitio de estudio, arroyo Paraíso, provincia de Misiones, Argentina. 1- Desembocadura del arroyo Paraíso en el rio Uruguay, 2 - Salto Paraíso, 7 km aguas arriba de la desembocadura.

Figure 1. Study site, Paraíso stream, Misiones province, Argentina. 1- Mouth of the Paraíso stream in the Uruguay River 2- Paraíso Fall, 7 km upstream to the stream's mouth.

gonadal teniendo en cuenta características macroscópicas de las gónadas como color, transparencia, vascularización superficial, flacidez y en el caso de ovarios, tamaño, posición en la cavidad abdominal y grado de visualización de los ovocitos (Vazzoler, 1996). La evaluación del llenado gástrico fue semicuantitativa y los estómagos fueron clasificados en llenos, semillenos y vacíos. Los estómagos llenos y semillenos se conservaron, debidamente rotulados, en formol al $10 \%$ para su posterior análisis en laboratorio.

La identificación de especies se realizó mediante el uso de claves, bibliografía y en algunos casos a través del asesoramiento de consultores externos especializados.

Se analizó la composición específica de las capturas. La diversidad alfa se expresó en número (riqueza de especies) y en diversidad "verdadera" en números efectivos. Esta se estimó utilizando la conversión del índice de Shannon-Wienner ( $\left.\mathrm{H}^{\prime}\right)$ propuesta por Jost (2006) por medio de la ecuación:

${ }^{q} D=\exp \left(H^{\prime}\right)$

donde ${ }^{a} D$ : diversidad en números efectivos, q: sensibilidad del índice a las abundancias relativas de las especies de la comunidad. En 
este caso se utilizó $q=1$, considerando que todas las especies son incluidas con un peso exactamente proporcional a su abundancia en la comunidad (Moreno et al., 2011).

Para inferir si los inventarios estaban completos se construyó la curva de acumulación de especies y se utilizaron estimadores no paramétricos de riqueza de especies Chao 1 calculado con el programa EstimateS 9.1.0 (Conwell, 2013).

Para estimar la estructura de tallas de las capturas se recopilaron datos de la bibliografía sobre longitud estándar máxima de cada especie a partir de Reis et al. (2003) y las descripciones originales de las especies. Esto fue posible porque la mayoría de los ejemplares capturados fueron adultos. Los valores hallados $(n=30)$ fueron organizados en clases, calculadas por medio de la fórmula de Sturges $(k=1+3,32$ $\log n$, siendo k el número de clases y $n$ el tamaño de la muestra). El tamaño del intervalo de las clases fue calculado a partir del cociente entre la amplitud de tamaño y el número de clases. La frecuencia de ocurrencia de las especies fue analizada siguiendo la fórmula:

$\% \mathrm{FO}=\mathrm{P} / \mathrm{Q} \times 100$

donde $\mathrm{FO} \%$ = frecuencia de ocurrencia porcentual de la especie, $P=$ número de muestras en las que la especie estuvo presente y $\mathrm{Q}=$ número total de muestras colectadas. Las especies fueron clasificadas según las categorías propuestas por Lasso (2001), en raras o accidentales (\%FO $<25 \%$ ), comunes o accesorias (\%FO> $25 \%$ y $<50 \%)$ y comunes o abundantes (\%FO> 50\%).

Se calculó el índice de dominancia comunitaria (IDC) de McNaughton, según la fórmula de Goulding et al. (1988):

$I D C=(Y 1+Y 2) / Y$

donde $Y 1+Y 2$ es la suma de la abundancia de las dos especies dominantes e $Y$ es la suma de la abundancia de todas las especies.

El contenido estomacal de 22 ejemplares pertenecientes a cinco especies fue tratado siguiendo la metodología propuesta por Prej y Colomine (1981). Se identificaron los ítems alimentarios bajo microscopio estereoscópico y para determinar las categorías tróficas de la comunidad íctica se siguió la clasificación planteada por Hahn et al. (1998). A los resultados obtenidos se sumaron datos de fuentes secundarias para definir los grupos funcionales de la comunidad.

La evaluación de la actividad reproductiva de los peces se realizó por medio de la distribución estacional de los estadios gonadales distinguiendo individuos en reproducción, aquellos clasificados como en maduración, maduro o en desove para distinguirlos de los ejemplares en reposo, que no presentaban signos de actividad reproductiva. Como signos de actividad reproductiva se consideró el aumento de tamaño de los ovarios y de los ovocitos, resultado de la vitelogénesis, pudiendo ser observados a simple vista. La flacidez de los ovarios, el aumento de la vascularización y la presencia de ovocitos maduros remanentes se consideraron como indicios de desove.

\section{RESULTADOS}

Los parámetros físicos y químicos registrados (Tabla 1) indican que el agua en la cuenca baja del arroyo Paraíso, se caracteriza por presentar $\mathrm{pH}$ neutro a levemente básico y oxigenación elevada. La temperatura del agua varió en relación con las condiciones ambientales y con características de los arroyos como presencia de vegetación marginal, pozas y rápidos. Se capturaron 217 individuos pertenecientes a 35 especies, distribuidas en cuatro órdenes y 11 familias (Tabla 2). La mayor abundancia correspondió al orden Characiformes con el 66\% de la captura seguido por los Siluriformes (19,5\%), Cichliformes (8\%) y Gymnotiformes $(6,9)$.

En el orden Characiformes, el mayor número de especies fue de la familia Characidae (seis especies), en el orden Siluriformes la familia Loricariidae (seis especies), en el orden Cichliformes la familia Cichlidae con siete especies mientras que el orden Gymnotiformes estuvo representado por una especie de la familia Sternopygida.

La diversidad verdadera de números efectivos fue de 16,44 . Con el esfuerzo de pesca realizado se logró un inventario del $69 \%$ de la riqueza específica estimada para el arroyo Paraíso. De acuerdo con el estimador no paramétrico de diversidad Chao 1, el valor máximo de riqueza de especie esperado es de 51 (Figura 2). Los resultados de la frecuencia de ocurrencia de especies indican que el $66 \%$ de las especies relevadas son raras o accidentales, con 
Tabla 1. Parámetros físicos y químicos del agua en el arroyo Paraíso, Misiones, Argentina.1: Desembocadura del arroyo Paraíso en el río Uruguay, 2: Salto Paraíso, OD: Oxígeno Disuelto.

Table 1. Physical and chemical parameters of water in the Paraíso stream, Misiones, Argentina. 1: Mouth of the Paraíso stream in the Uruguay River, 2: Paraíso Fall, OD: dissolved oxygen.

\begin{tabular}{ccccccc}
\hline & Sitio & $\mathrm{pH}$ & $\begin{array}{c}\mathrm{OD} \\
(\mathrm{ppm})\end{array}$ & $\begin{array}{c}\text { Conductividad } \\
(\mu \mathrm{S} / \mathrm{cm})\end{array}$ & $\begin{array}{c}\text { Temperatura } \\
\text { Agua }\left({ }^{\circ} \mathrm{C}\right)\end{array}$ & $\begin{array}{c}\text { Temperatura } \\
\text { ambiente }\left({ }^{\circ} \mathrm{C}\right)\end{array}$ \\
\hline \multirow{2}{*}{ Primavera } & 1 & 7,66 & 7,66 & 32 & 19.8 & 19,5 \\
& 2 & 7,66 & 7,66 & 32 & 17,5 & 18 \\
Verano & 1 & 7,9 & 9,6 & 38 & 19 & 25,5 \\
& 2 & 8,27 & 9,6 & 38 & 19 & 26 \\
Otoño & 1 & 7,3 & 7,54 & 40 & 28,5 & 31 \\
& 2 & 7,5 & 7,54 & 31,32 & 26 & 29 \\
Invierno & 1 & 7,46 & 7,4 & 15 & 20,5 & 27 \\
& 2 & 7 & 7,4 & 15 & 19 & 25 \\
\hline
\end{tabular}

abundancia relativa inferior al $10 \%$. Cuatro especies son comunes: Crenicichla missioneira, Leporinus amae, Astyanax lacustris y Leporinus striatus. Siete especies que representan el $19 \%$ de las identificadas, son endémicas de la cuenca del río Uruguay: Astyanax paris, $A$.
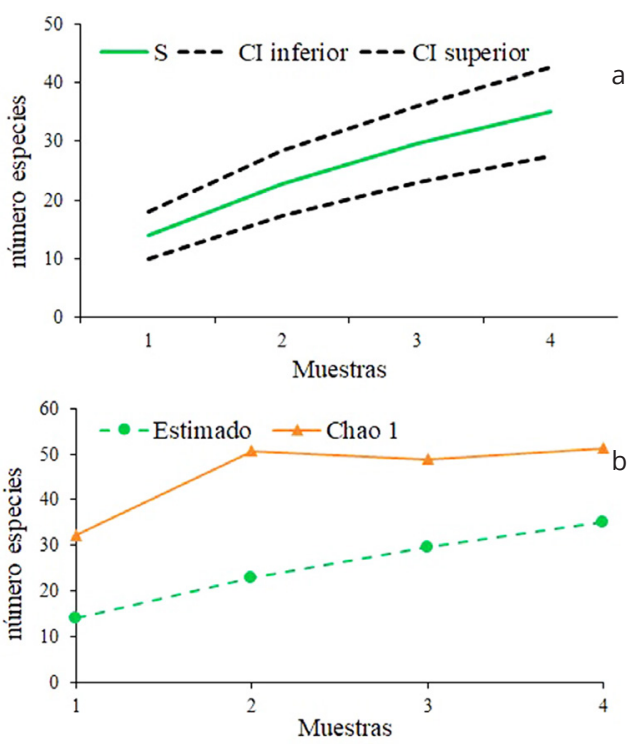

Figura 2. a. Curva de acumulación de las especies ícticas en el arroyo Paraíso. Misiones, Argentina. (S: estimado, Cl: intervalo de confianza) b. Estimador no paramétrico de la riqueza de especies Chao 1.

Figure 2. a. Accumulation curve of fish species in the Paraiso Stream, Misiones, Argentina. (S: estimated, $\mathrm{Cl}$ : confidence interval) b. Chao 1 non parametric estimator of species richness. troya, Crenicichla celidochilus, C. tendybaguassu, C. missioneira, Leporinus amae y Hemiancistrus fulliginosus (Tabla 2).

La dominancia comunitaria varió a lo largo de las estaciones del año, alcanzando su mayor valor en primavera, con dos especies que representan el $17 \%$ de la abundancia relativa (Tabla 3).

La talla máxima registrada en la bibliografía para las especies colectadas varió entre $8,2 \mathrm{~cm}$ y $80 \mathrm{~cm}$ de longitud estándar, con un valor de mediana de 19,9 cm (desvío estándar: 17,1). Se definieron, por medio de la fórmula de Sturges, seis clases de longitud estándar con un intervalo (i) de $13 \mathrm{~cm}$. La gran mayoría de las especies (83\%) estuvieron incluidas en la primera clase (i: $8-21 \mathrm{~cm}$ ) y la segunda clase (i: 21,1 y $34 \mathrm{~cm}$ ) y son consideradas de pequeño porte. El resto de las especies (17\%) estuvieron comprendidas en las clases restantes y son de medio a gran porte (Figura 3).

Del total de la captura, el $80 \%$ presentó estómagos vacíos y del análisis de 22 estómagos Ilenos y/o semillenos de ejemplares de Apareiodon piracicabae, Iheringichthys labrosus, L. amae, Steindachnerina biornata y L. striatus se destaca la importancia de las fases larvales de insectos terrestres de los órdenes Trichoptera y Diptera y de materia orgánica proveniente de la vegetación riparia (Tabla 4).

Con los resultados obtenidos en este trabajo y de fuentes secundarias, se establecieron seis categorías tróficas, con un elevado porcentaje de especies incluidas en las categorías detritívoro y 
Tabla 2. Lista de especies registradas en el arroyo Paraíso, Misiones, Argentina. AR: abundancia relativa, \%FO: frecuencia de ocurrencia, Co: especie común o abundante, Ac: especie accesoria, Ad: especie accidental, * Especie endémica de la región.

Table 2. List of species registered in the Paraíso Stream, Misiones, Argentina. AR: relative abundance, \%FO: frequency of occurrence, Co: common or abundant species, Ac: accessory species, Ad: accidental species, * Endemic species of the region.

\begin{tabular}{lcc}
\hline \multicolumn{1}{c}{ ESPECIES } & AR & $\% \mathrm{FO}$ \\
\hline $\begin{array}{l}\text { Orden Characiformes } \\
\text { Familia Parodontidae }\end{array}$ & & \\
Apareiodon piracicabae (Eingenmann, 1907) & 6,02 & $\mathrm{Ac}$ \\
Familia Anostomidae & & \\
Schizodon nasutus Kner, 1858 & 1,85 & $\mathrm{Ad}$ \\
Leporinus acutidens (Valenciennes, 1837) & 0,93 & $\mathrm{Ad}$ \\
Leporinus striatus Kner, 1858 & 11,67 & $\mathrm{Co}$ \\
Leporinus amae Godoy, 1980* & 19,91 & $\mathrm{Co}$ \\
Familia Characidae & & \\
Astyanax paris Azpelicueta, Almirón \& Casciotta, 2002* & 0,46 & $\mathrm{Ad}$ \\
Astyanax pynandi Azpelicueta, Almirón, Bechara, Roux \& Ruiz Diaz, 2003 & 0,93 & $\mathrm{Ad}$ \\
Astyanax lacustris (Lütken, 1875) & 1,85 & $\mathrm{Co}$ \\
Astyanax cf lacustris & 0,46 & $\mathrm{Ad}$ \\
Astyanax troya Azpelicueta, Almirón \& Casciotta, 2002* & 0,93 & $\mathrm{Ad}$ \\
Acestrorhynchus pantaneiro Menezes, 1992 & 5,09 & $\mathrm{Ac}$ \\
Familia Cynodontidae & & \\
Rhaphiodon vulpinus Spix \&Agassiz, 1829 & 0,46 & $\mathrm{Ad}$ \\
Familia Prochilodontidae & & \\
Prochilodus lineatus (Valenciennes, 1837) & & \\
Familia Curimatidae & 0,93 & Ac \\
Steindachnerina biornata (Braga \& Azpelicueta, 1987) & & \\
Steindachnerina brevipinna (Eingenmann \& Eingenmann, 1889) & 12,50 & Ac \\
Familia Erythrinidae & 1,39 & Ad \\
Hoplias sp. & & \\
& 0,93 & Ac
\end{tabular}

\section{ORDEN SILURIFORMES}

Familia Pimelodidae

Iheringichthys labrosus (Lütken, 1874)

Pimelodus absconditus Azpelicueta, 1995

Pimelodus sp.

Sorubin lima (Bloch \& Schneider, 1801)

\section{Familia Loricariidae}

Lorycariichthys platymetopon Isbrücker \& Nijssen, 1979

Hypostomus isbrueckeri Reis, Weber \& Malabarba, 1990

Familia Heptateridae

Pimelodella gracilis (Valenciennes, 1835)

\section{ORDEN CICHLIFORMES}

\section{Familia Cichlidae}

Crenicichla celidochilus Casciotta, 1987*

\section{ORDEN GYMNOTIFORMES}


Tabla 3. Índice de dominancia comunitaria (IDC) por estación del año en el arroyo Paraíso, Misiones, Argentina.

Table 3. Community dominance index (IDC) according to the season of the year in the Paraiso Stream of the year. Paraíso Stream, Misiones, Argentina.

\begin{tabular}{|c|c|c|}
\hline & IDC & Especies Dominantes \\
\hline \multirow[t]{2}{*}{ Primavera } & 0,81 & Leporinus striatus \\
\hline & & Apareiodon piracicabae \\
\hline \multirow[t]{2}{*}{ Verano } & 0,42 & Steindachnerina \\
\hline & & Iheringichthys labrosus \\
\hline \multirow[t]{2}{*}{ Otoño } & 0,40 & Leporinus striatus \\
\hline & & Schizodon nasutus \\
\hline \multirow[t]{2}{*}{ Invierno } & 0,48 & Leporinus amae \\
\hline & & Leporinus striatus \\
\hline
\end{tabular}

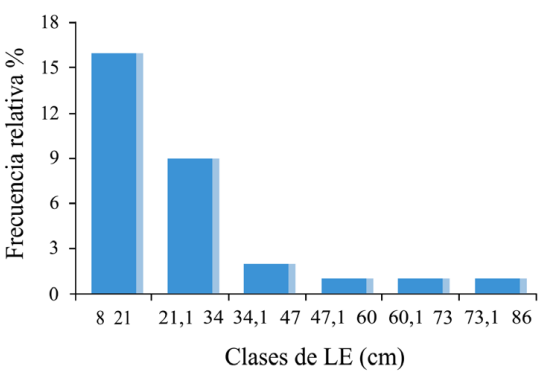

Figura 3. Distribución por intervalos de talla corporal de la comunidad íctica Arroyo Paraíso. LE: longitud estándar.

Figure 3. Body size distribution of fish community in the Paraíso Stream, Misiones, Argemtima (LE: standard length).

Tabla 4. Ítems alimentarios de peces en el Arroyo Paraíso, Misiones, Argentina. (Le: longitud estándar, n: número de estómagos analizados. El ítem insectos comprende: Larvas y pupas de los órdenes Trichoptera y Diptera (Chironomidae). RTVD: Restos de detrito vegetal en degradación, sedimento: barro, arena y piedra).

Table 4. Fish food items in Paraiso Stream, Misiones, Argentina. (Le: standard length, n: number of analyzed stomachs. The insect item includes: larvae and pupae of the Trichoptera and Diptera (Chironomidae) orders. RTVD: Vegetable detritus in degradation, sediment: mud, sand and stone).

\begin{tabular}{|c|c|c|c|}
\hline Especies & $\mathbf{n}$ & Le $(\mathrm{cm})$ & Ítems alimentarios \\
\hline Apareiodon piracicabae & 1 & 17 & Odonata, Sedimento, detrito orgánico. \\
\hline Iheringichthys labrosus & 2 & $13,5-16,5$ & $\begin{array}{l}\text { RTVD, Insectos (larvas de Chironomidae, } \\
\text { Ceratopogonidae, ninfas de Odonata y pupas } \\
\text { de Diptera) Gasterópodos }\end{array}$ \\
\hline Leporinus amae & 3 & $12-14$ & $\begin{array}{l}\text { RTVD, Sedimento, Insectos (larvas de } \\
\text { Ceratopogonidae, Trichoptera y ninfas de } \\
\text { Odonata) }\end{array}$ \\
\hline Steindachnerina biornata & 6 & $8-40$ & Detritos orgánicos \\
\hline Leporinus striatus & 10 & $11,5-14$ & $\begin{array}{l}\text { RTVD, detritos orgánicos, larvas de insectos } \\
\text { (Diptera: Ceratopogonidae y Chironomidae, } \\
\text { Trichoptera, Odonata, Ephemeroptera, } \\
\text { Hymenoptera) y pupas de Diptera. }\end{array}$ \\
\hline
\end{tabular}

omnívoro (Tabla 5).

El $87 \%$ de los ejemplares sexados, fueron hembras de especies como $A$. piracicabae, $L$. striatus, Acestrorhynchus pantaneiro, Prochilodus lineatus, Hoplias sp., S. biornata, Rhaphiodom vulpinus, I. labrosus y Pimelodus absconditus, de las cuales el 57\% presentó signos de madurez para el desove. Durante la primavera y el invierno se detectó la mayor actividad reproductiva, con la presencia de hembras maduras de $L$. striatus y A. piracicabae. En la época de otoño la actividad reproductiva incluyó a ejemplares en desove de $L$. striatus. Los individuos en reposo fueron los que predominaron durante el verano y la actividad reproductiva en esta estación estuvo relacionada con individuos maduros de $S$. biornata (Figura 4).

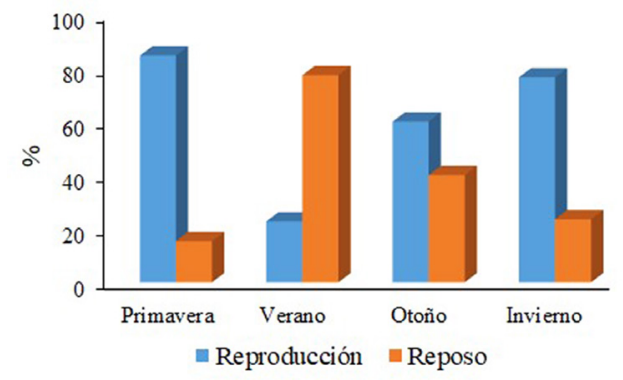

Figura 4. Actividad reproductiva de la comunidad íctica del arroyo Paraíso, Misiones, Argentina.

Figure 4. Reproductive activity of the fish community in Paraíso Stream, Misiones, Argentina. 
Tabla 5. Categoría trófica de las especies identificadas en el arroyo Paraíso.1. Flores et al. (2015), 2. Vazzoler et al. (1997), 3. Monasterio de Gonzo et al. (2003), 4. Sverlij et al. (1998), 5. Casciotta et al. (2005), 6. Alves da Silva et al. (2012), 7. De Lucena \& Kullander (1992),

8. Ross et al. (1992), 9. Hahn et al. (1998), 10. Hahn et al. (2002), 11. Masdeu et al. (2011).

Table 5. Trophic category of the identified species in the Paraíso stream. 1. Flores et al. (2015), 2. Vazzoler et al. (1997), 3. Monasterio de Gonzo et al. (2003), 4. Sverlij et al. (1998), 5. Casciotta et al. (2005), 6. Alves da Silva et al. (2012), 7. de Lucena \& Kullander (1992),

8. Ross et al. (1992), 9. Hahn et al. (1998), 10. Hahn et al. (2002), 11. Masdeu et al. (2011).

\begin{tabular}{|c|c|}
\hline $\begin{array}{l}\text { Categorías } \\
\text { tróficas }\end{array}$ & Especies \\
\hline Piscívoros & $\begin{array}{l}\text { Acestrorhynchus pantaneiro (1) } \\
\text { Rhaphiodon vulpinus (2) } \\
\text { Sorubin lima (10) } \\
\text { Hoplias malabaricus (2) } \\
\text { Crenicichla vittata (1) }\end{array}$ \\
\hline Omnívoros & $\begin{array}{l}\text { Astyanax lacustris (3) } \\
\text { Astyanax lacustris (6) } \\
\text { Pimelodus absconditus (1) } \\
\text { Pimelodella gracilis (5) } \\
\text { Crenicichla misionneira (11) } \\
\text { Leporinus acutidens (9) } \\
\text { Apareiodon piracicabae } \\
\text { Leporinus amae } \\
\text { Leporinus striatus }\end{array}$ \\
\hline Detritívoros & $\begin{array}{l}\text { Hemiancistrus fuliginosus (8) } \\
\text { Steindachnerina brevipina (1) } \\
\text { Hypostomus luteomaculatus (4) } \\
\text { Prochilodus lineatus (4) } \\
\text { Hypostomus isbrueckeri (8) } \\
\text { Loricariichthys platymetopon (10) } \\
\text { Steindachnerina biornata }\end{array}$ \\
\hline $\begin{array}{c}\text { Detritivoro con } \\
\text { tendencia a la } \\
\text { herbivoría }\end{array}$ & $\begin{array}{l}\text { Astyanax paris (1) } \\
\text { Schizodon nassutus (1) }\end{array}$ \\
\hline Insectívoros & $\begin{array}{l}\text { Sternopygus macrurus (10) } \\
\text { Crenicichla tendybaguazu (11) }\end{array}$ \\
\hline Bentófagos & Iheringichthys labrosus (11) \\
\hline
\end{tabular}

\section{DISCUSIÓN}

Los datos aportados por este trabajo contribuyen a la información existente y permiten reforzar la idea sobre la elevada diversidad íctica en las aguas interiores de la provincia de Misiones. La composición responde a los patrones característicos de la ictiofauna Neotropical, donde los órdenes registrados concentran la mayor diversidad taxonómica y abundancia, con especies que ocupan los más diversos ambientes y han experimentado la radiación adaptativa más importante en los sistemas fluviales de Sudamérica (Reis et al., 2016; Giraudo, 2017). El elevado número de especies registrado en el arroyo estudiado es comparable a otros tributarios del río Uruguay tales como Quaraí Chico (Castro-Pessano et al., 2005), Santa María (Bossemeyer et al., 1985), Jaguarí y Cambará (Copatti y Copatti, 2011; Copatti et al., 2009) y Yabotí (Flores et al., 2015). Al expresar la diversidad en número de especies efectivas se obtuvo un valor elevado ( ${ }^{1} \mathrm{D}$ : 16,44 especies efectivas) en comparación con los datos reportados por Scipioni et al. (2016) para la comunidad de peces en el río Paraná en ambientes asociados a la represa hidroeléctrica Yacyretá, '1D: 14,01 especies efectivas en Itá Ibaté, provincia de Corrientes.

La alta diversidad íctica de los cursos de agua de Misiones se relaciona con los patrones de distribución natural de las especies, asociada en cada caso, a diversos factores tales como los geográficos, ambientales y atributos biológicos, ecológicos y evolutivos, los que en conjunto modelan la estructura y dinámica de la comunidad. Este territorio cuenta con la más alta tasa de endemismo de todas las regiones ícticas de la Argentina (López et al., 2005). Las especies endémicas son importantes en aspectos de conservación debido a que, por tener distribuciones muy restringidas, pueden ser más fácil e irreversiblemente afectadas por las actividades humanas, que pueden destruir sus hábitats o ser fácilmente sobreexplotadas (Giraudo et al., 2012). En esta investigación se registraron siete especies endémicas de la cuenca del río Uruguay Astyanax paris, A. troya, C. celidochilus, C. missioneira, C. tendybaguassu, $H$. fulliginosus y L. amae. Se suman a otras especies endémicas citadas para el arroyo Paraíso, tales como Rineloricaria reisi Ghazzi, 2008 (Almirón et al., 2014) y Australoheros ykeregua Říčan, Piálek, Almirón \& Casciotta, 2011(Říčan et al., 2011).

El estimador de la completitud del inventario de especies (Chao 1), indicó que estaría faltando reconocer al menos un 30\% de especies de peces del arroyo Paraíso. Con muestreos más extensivos e intensivos se podría incrementar la riqueza de especies, quedando aún por explorar la cuenca media-alta. 
Trabajos de investigación sugieren que la mayoría de las comunidades animales se caracterizan por presentar pocas especies abundantes y muchas especies representadas por pocos individuos (Matthews, 1998). Los resultados de nuestro trabajo coinciden con esta afirmación, donde el $66 \%$ de especies estuvieron incluidas en la categoría raras o accidentales y cuatro especies fueron comunes L. striatus, L. amae, A. lacustris y C. missioneira. Esta estructura comunitaria, podría variar en relación con la capacidad de explotación de los recursos presentes en el biotopo en diferentes momentos y dependiendo de la fase del ciclo de vida (Lemes y Garutti, 2002). La estimación de estos atributos de la estructura de la comunidad íctica del arroyo Paraíso permite comprender el comportamiento de esta comunidad y aporta información para la administración, manejo y conservación del recurso. En este sentido el índice de dominancia que registró un máximo en primavera $(0,81)$, estuvo dado por ejemplares de L. striatus y A. piracicabae en correspondencia con el registro de individuos en reproducción.

Los arroyos tributarios a los grandes ríos de la Cuenca del Plata, en este caso el Uruguay, muestran características propias, como la presencia de barreras físicas naturales, como planchas de piedras y caídas (cascadas), diferentes sustratos y niveles de profundidad, contribuyendo a procesos de especiación por aislamiento de poblaciones de especies de pequeño porte en microhábitats. Más del $60 \%$ de las especies que integran la comunidad íctica del arroyo Paraíso tienen longitud estándar promedio menor a $15 \mathrm{~cm}$, lo que permite asumir la dominancia de especies de pequeño porte, característica que se repite en los arroyos de Misiones (Araya et al., 2012; Flores et al., 2009) y es un patrón común en tributarios a los grandes ríos de la Cuenca del Plata (Castro et al., 2003). En los sistemas fluviales de Misiones, la vegetación marginal constituye una franja muy angosta formando una selva en galería a lo largo de los ríos, que incluye muchos árboles de la selva climáxica misionera y varias especies de los géneros Inga, Pouteria (mata ojos) y Sapium (lecherones, curupíes), entre otras (Rodríguez et al., 2005). Esta vegetación contribuye a la estructura de la comunidad íctica al representar un recurso alimentario y condiciones favorables para el desove y el progreso de las primeras etapas del desarrollo.

El paisaje de la cuenca del arroyo Paraíso se presenta fragmentado con diferente grado de degradación, relacionado con las principales actividades productivas de la zona que incluyen cultivos de aromáticas como citronella, tabaco, citrus y frutas tropicales (Ministerio de Agroindustria, 2016). La eliminación de la vegetación marginal impacta negativamente sobre la ictiofauna, tanto en la alimentación como en la reproducción (Menezes et al., 2007). Los resultados del presente trabajo muestran que uno de los recursos alimentarios más utilizados por las especies analizadas fueron los insectos acuáticos o terrestres en distintas etapas de desarrollo que juegan un rol significativo en la alimentación, así como la materia orgánica y detritos por su contenido en microorganismos. Se ha demostrado la importancia del material orgánico alóctono importado desde la vegetación marginal en la dieta de los peces (Lowe-McConnell, 1999; Sabino \& Corrêa e Castro, 1990; Menezes et al., 2007).

Los peces detritívoros fueron predominantes en la comunidad del arroyo Paraíso, destacándose S. biornata y Loricariichthys platymetopon como los más abundantes y por su papel en la recirculación de los nutrientes al utilizar todo tipo de materia orgánica particulada. Algunos detritívoros de pequeño porte como A. paris y Schizodon nassutus presentaron tendencia a la herbivoría, lo que hace suponer que esta modificación alimentaria depende de la cantidad de material vegetal de origen alóctono que ingrese al cuerpo de agua. La categoría de los omnívoros incluye especies generalistas como P. absconditus, Pimelodella gracilis, C. misionneira y Leporinus acutidens que aprovechan los recursos más abundantes dentro del ecosistema acuático, reflejando esa plasticidad trófica bien conocida en los peces neotropicales (Abelha et al., 2001).

La actividad reproductiva de la comunidad íctica se evidenció durante todas las estaciones del año, en especies como L. striatus, A. piracicabae y $S$. biornata, que se caracterizan por tener 
estrategia reproductiva del tipo migrador de corta distancia o no migradores, con fecundación externa y sin cuidado parental (Agostinho et al., 2004). Se ha comprobado que en el arroyo Yabotí, inmerso en la RBY, L. striatus presentó desarrollo sincrónico de los ovocitos lo que permite desoves parcelados que exponen a la descendencia a diferentes condiciones ambientales favoreciendo su reproducción en diferentes momentos del año (Araya et al., 2018). Se concluye que la ictiofauna del arroyo Paraíso tiene una elevada riqueza de especie con presencia de muchos endemismos, altamente dependiente del material orgánico alóctono para la alimentación, es por ello que se hace imprescindible que se apliquen las leyes vigentes de protección y conservación de los cursos de agua y de la fauna íctica en la provincia de Misiones, tal como la Ley de "Bosques Protectores" que protege a todas aquellas formaciones boscosas que se encuentran en una faja correspondiente a tres veces el ancho del cauce y no menor a $5 \mathrm{~m}$ a cada orilla de los arroyos (Ley Prov. 3426).

\section{REFERENCIAS}

Abelha, M. C. F., Agostinho, A. A. \& Goulart, E. (2001). Plasticidade trófica em peixes de água doce. Acta Scientiarum, 23(2): 425-434.

Agostinho, A., Gomes, L., Veríssimo, S. \& Okada, E. (2004). Flood regime and fish: effects on spawning, recruitment and attributes of the assemblages in the upper Paraná River floodplain. Reviews in Fish Biology and Fisheries, 14: 11-19.

Araya, P., Hirt, L. y Flores, S. (2012). Composición y abundancia de la comunidad íctica de la Reserva Biosfera Yabotí. Arroyo Yabotí. Misiones. Argentina. AquaTIC, 36: 21-27.

Araya, P., Flores, S. y Hirt, L. (2018). Ecología reproductiva y crecimiento de Leporinus striatus en un afluente del río Uruguay. Misiones Argentina. AquaTIC, 50: 1-12.

Almirón, A., Casciotta, J., Piálek, L., Ruiz Díaz, F. \& Říčan, O. (2014). First record of Rineloricaria reisi, R. stellata and R. zaina (Siluriformes: Loricariidae) from the Río Uruguay basin in Argentina. Check List, 10(6): 1528-1530.
Almirón, A., Casciotta, J., Piálek, L., Doubnerová, K. \& Říčan, O. (2015). Oligosarcus amome (Ostariophysi: Characidae), a new species from the río Uruguay basin, Misiones, Argentina. Zootaxa, 3915 (1): 581-590.

Alves da Silva, D., Rodrigues Pessoa, E., Almeida, S., Chellappa, N. \& Chellappa, S. (2012). Ecologia alimentar de Astyanax lacustris (Osteichthyes: Characidae) na Lagoa do Piató, Assu, Rio Grande do Norte, Brasil. Biota Amazonia, 2: 74-82.

Bossemeyer, I. M., Weis, M. L. C., Benemann, S. T. \& Bier, M. L. S. (1985). Ictiofauna do rio Santa Maria, RS. Ciencias Naturales, 7: 209-222.

Cabrera, A. y Wilink, A. (1973). Biogeografía de América Latina. Monografía 13, Serie Biología. Washington DC: Programa Regional de Desarrollo Científico y Tecnológico.

Casciotta, J., Almirón, A. y Bechara, J. (2005). Peces del Iberá - hábitat y diversidad. La Plata: Grafikar. Fundación Ecos, UNLP y UNNE.

Casciotta, J., Almirón, A., Piálek, L. \& Říčan, O. (2012). Cyanocharax obi, a new species (Characiformes: Characidae) and the first record of the genus from tributaries of the river Paraná basin, Argentina. Zootaxa, 3391:39-51.

Castro, R., Casatti, L., Santos, H., Ferreira, K., Ribeiro, A., Benine, R., Dardis, G., Melo, A., Stopiglia, R., Abreu, T., Bockmann, F., Carvalho, M., Gibran, F. \& Lima, F. (2003). Estrutura e composição da ictiofauna de riachos do Rio Paranapanema, Sudeste e Sul do Brasil. Biota Neotropica, 3(1): 1-31.

Castro-Pessano, E., de Oliveira Azevedo, C., Morini Querol, M., Querol, E., Gonçalves, L. Bortoluzzi Castro, L., Bortoluzzi Pinto, T. \& Corrêa, F. (2005). Ictiofauna do arroio Quarai-Chico, bacia do medio rio Uruguai, no interior do Parque Estadual do Espinilho, Rio Grande do Sul, Brasil. Biotemas, 18: 143-153.

Conwell, R. (2013). Estimates: Statistical estimation of species richness and shared species for samples. Versión 9.1.0.

Copatti, E. \& Copatti, B. (2011). ). Variacão sazonal e diversidade de peixes do rio Cambará, bacia do rio Uruguai. Biota Neotropica, 11: 265-271. 
Copatti, C., Gonçalves-Zanini, L. \& Valente, A. (2009). Ictiofauna da microbacia do rio Jaguari, Juaguari/RS, Brasil. Biota Neotropica, 9: 179-186.

de Lucena, C. A. \& Kullander, S. O. (1992). The Crenicichla (Teleostei: Cichlidae) species of the Uruguai River drainage in Brazil. Ichthyological Exploration of Freshwaters, 3: 97-192.

Fontana, J. L. (2014). La vegetación reófila del Nordeste argentino. Las comunidades vegetales con Podostemaceae de la Provincia de Misiones. Boletín de la Sociedad Argentina de Botánica, 49: 115-136.

Flores, S., Araya, P. \& Hirt, L. (2009). Fish diversity and community structure in a tributary stream of the Paraná River. Acta Limnologica Brasiliensia, 1 (1): 57-66

Flores, S., Hirt, L. y Araya, P. (2015). Estructura y dinámica de la comunidad íctica del arroyo Yabotí, Reserva de Biosfera Yabotí, Misiones, Argentina. Revista Mexicana de Biodiversidad, 86: 386-395

Giraudo, A., Povedano, H., Belgrano, M.J., Pardiñas, U.J., Miquelarena A.M. \& Ligier, D. (2003). Biodiversity status of the interior Atlantic forest of Argentina. In: Galindo-Leal, C., de Gusmao-Camara, I. (Eds.), The Atlantic forest of South America: Biodiversity status, threats, and outlook (pp. 160-180). Island Press: Washington, D. C.

Giraudo, A. (2017). Región Humedales Misioneros. En: Benzaquen, L., Blanco, D., Bo, R., Kandus, P, Lingua, G. y Minotti, P. (Eds.). Regiones de Humedales de Argentina (pp. 93110). Ministerio de Ambiente y Desarrollo Sustentable, Fundación Humedales/Wetlands International, Universidad Nacional de San Martín y Universidad de Buenos Aires.

Gómez, S. y Chebez, J. C. (1996). Peces de la Provincia de Misiones. En: Fauna Misionera, Catálogo de los vertebrados de la provincia de Misiones (Argentina) (pp. 38-70). Buenos Aires: Literature of Latin America.

Goulding, M., Leal-Carvalho, M. \& Ferreira, E. (1988). Río Negro, rich life in poor water: amazonian diversity and food chain ecology as seen through fish communities. The Hague: SPB Academic Publishing.
Hahn, N. S., Agostinho, A. A., Gomes, L. C., \& Bini, L. M. (1998). Estructura trófica da ictiofauna do reservatório de Itaipu (Paraná-Brasil) nos primeiros anos de sua formação. Interciencia, 23: 299-305.

Hahn, N. S., Fugi, R., Peretti, D., Russo, M. R., \& Loureiro-Crippa, V. E. (2002). Estrutura trófica da ictiofauna da planície de inundação do alto rio Paraná. In: Agostinho, A. A., Thomaz, S. M., Rodrigues, L. \& Gomes, L. C. (Eds.), A Planicie de Inundação do Alto rio Paraná (pp. 123-126). Maringá: Area de Pesquisas Ecológicas de Longa Duração, Núcleo de Pesquisas em Limnologia, Ictiologia e Aqüicultura-Nupelia. Universidade Estadual de Maringá.

Jost, L. (2006). Entropy and diversity. Oikos, 113: 363-375.

Lasso, C. A. (2001). Los peces del alto río Negro, Amazonía boliviana: composición y consideraciones ecológicas y biogeográficas. Interciencia, 26: 236-243.

Lemes, E.M. \& Garutti, V. (2002). Ecologia da ictiofauna de um córrego de cabeceira da bacia do alto rio Paraná, Brasil. Iheringia Série Zoologia, 92(3): 69-78.

López, H. L., Miquelarena, A. M. y Ponte Gómez J. (2005). Biodiversidad y distribución de la ictiofauna mesopotámica. Temas de la biodiversidad del litoral fluvial argentino. Miscelanea, 14: 311-354.

Lowe-Mc Connel, R. H. (1999). Estudos ecológicos de comunidades de peixes tropicais. São Paulo: Editora da Universidade de São Paulo.

Margalot, J. (1985). Geografía de Misiones. Buenos Aires: Cultural Argentina.

Masdeu, M., Mello, F. T. D., Loureiro, M., \& Arim, M. (2011). Feeding habits and morphometry of Iheringichthys labrosus (Lütken, 1874) in the Uruguay River (Uruguay). Neotropical Ichthyology, 9: 657-664.

Matthews, W. J. (1998). Patterns in Freshwater Fish Ecology. New York: Chapman \& Hall. 
Menezes, N. A., Weitzman, S. H., Oyakawa, O. T., Lima, F. C. T., Castro, R. M. C. \& Weitzman, M.J. (2007). Peixes de água doce da Mata Atlântica: lista preliminar das espécies de água doce neotropicais. São Paulo: Museu de Zoologia - Universidade de São Paulo.

Ministerio de Agroindustria (2016). Proyecto de inclusión socioeconómica en áreas rurales. Ministerio del Agro y la Producción. Provincia de Misiones.

Moreno, C. E., Barragan, F., Pineda E. y Pavón N. P. (2011). Reanálisis de la diversidad alfa: alternativas para interpretar y comparar información sobre comunidades ecológicas. Revista Mexicana de Biodiversidad, 82: 1249-1261.

Monasterio de Gonzo, G., Barros, S, Mamaní, O. y Mosqueira, M. (2003). Ictiofauna del área noroeste de la cuenca Parano-Platense. Natura Neotropicalis, 36: 21-33.

Piálek, L., Říčan, O., Casciotta, J. R. \& Almirón, A. E. (2010). Crenicichla hu, a new species of cichlid fish (Teleostei: Cichlidae) from the Paraná basin in Misiones, Argentina. Zootaxa, 2537: 33-46.

Piálek, L., Dragová, K., Casciotta, J., Almirón, A. \& Říčan, O. (2015). Description of two new species of Crenicichla (Teleostei: Cichlidae) from the Lower Iguazú River with a taxonomic reappraisal of C. iguassuensis, C. tesay and C. yaha. Historia Natural, 3 (2): 5-27.

Prej, A. y Colomine, G. (1981). Métodos para el estudio de los alimentos y las relaciones tróficas de los peces. Caracas: Universidad Central de Venezuela; Polonia: Universidad de Varsovia.

Reis, R. E., Kullander, S. O. \& Ferraris Jr., C. J. (2003). Check list of the freshwater fishes of South Central and America. Porto Alegre: EDIPURCS.

Reis, R., Albert, J., Di Dario, F., Mincarone, M., Petry, P. \& Rocha, L. (2016). Fish biodiversity and conservation in South America. Journal of Fish Biology, 89: 12-47.

Říčan, O., Piálek, L., Almirón, A. \& Casciotta, J. (2011). Two new species of Australoheros (Teleostei: Cichlidae), with notes on diversity of the genus and biogeography of the Río de la Plata basin. Zootaxa, 2982: 1-26.
Roa, B. H. (2005). Lista de las especies de peces del tramo Ituzaingó-Iguazú del río Alto Paraná (Argentina). Revista de Ciencia y Tecnología, 7:73-79.

Rodríguez, M., Cardozo A, Ruiz Díaz M. y Prado D. (2005). Los bosques nativos misioneros: estado actual de su conocimiento y perspectivas. En: Arturi, F.M., Frangi, J.L, y Goya, J.F. (Eds) Ecología y manejo de los bosques de Argentina (pp. 3-33). La Plata: EDULP. Recuperado en: http://hdl. handle.net/10915/15915. (fecha de acceso: 27-12-2019).

Ross, B., Fagundes, L. S., \& Copatti, C. E. (1992). Peixes do Rio Cambará. Seminário Interinstitucional de Ensino Pesquisa e Extensão. Universidade de Cruz Alta.

Rosso, J., Mabragaña, E., Avigliano, E., Schenone, N. \& Díaz de Astarloa, J. M. (2013). Short spatial and temporal scale patterns of fish assemblages in a subtropical rainforest mountain stream. Studies on Neotropical Fauna and Environment, 48: 199-209.

Sabino, J. \& Corrêa e Castro, M. C. R. (1990). Alimentação, período de atividade e distribuição espacial dos peixes de um riacho da floresta Atlântica (Sudeste do Brasil). Revista Brasileira de Biología, 50(1): 23-36.

Scipioni, N., Casciotta, J. R., Almirón, A. E., Santinón, J. J. y Ruiz-Díaz, F. J. (2016). Análisis de la diversidad de peces en ambientes asociados al río Paraná en el área de la represa de Yacyretá. Revista Veterinaria, 27 (2): 86-92.

Sverlij, S. B., Delfino, L. R., López, H. L. y EspinachRos, A. (1998). Peces del río Uruguay. Guía ilustrada de las especies más comunes del río Uruguay inferior y el embalse de Salto Grande. Paysandú: Publicaciones de la Comisión Administradora del río Uruguay.

Vazzoler, A. (1996). Biologia da reprodução de peixes teleósteos: teoría e pratica. Maringá: EDUEM.

Vazzoler, A., Agostinho, A. \& Hann, N. (1997). A Planície de Inundacão do Alto Paraná. Aspectos físicos, biológicos e socioeconômicos. Maringá: Nupelia. 